\title{
The integrated Sachs-Wolfe imprint of cosmic superstructures: a problem for $\Lambda$ CDM
}

\author{
Seshadri Nadathur, ${ }^{a, b}$ Shaun Hotchkiss ${ }^{c}$ and Subir Sarkar ${ }^{a}$ \\ ${ }^{a}$ Rudolf Peierls Centre for Theoretical Physics, University of Oxford, Oxford OX1 3NP, UK \\ ${ }^{b}$ Fakultät für Physik, Universität Bielefeld, Postfach 100131, 33501 Bielefeld, Germany \\ ${ }^{c}$ Department of Physics, University of Helsinki and Helsinki Institute of Physics, P.O. Box \\ 64, FIN-00014 University of Helsinki, Finland \\ E-mail: seshadri@physik.uni-bielefeld.de, shaun.hotchkiss@helsinki.fi, \\ s.sarkar@physics.ox.ac.uk
}

\begin{abstract}
A crucial diagnostic of the $\Lambda \mathrm{CDM}$ cosmological model is the integrated SachsWolfe (ISW) effect of large-scale structure on the cosmic microwave background (CMB). The ISW imprint of superstructures of size $\sim 100 h^{-1} \mathrm{Mpc}$ at redshift $z \sim 0.5$ has been detected with $>4 \sigma$ significance, however it has been noted that the signal is much larger than expected. We revisit the calculation using linear theory predictions in $\Lambda$ CDM cosmology for the number density of superstructures and their radial density profile, and take possible selection effects into account. While our expected signal is larger than previous estimates, it is still inconsistent by $>3 \sigma$ with the observation. If the observed signal is indeed due to the ISW effect then huge, extremely underdense voids are far more common in the observed universe than predicted by $\Lambda \mathrm{CDM}$.
\end{abstract}

Keywords: integrated Sachs-Wolfe effect, cosmological parameters from CMBR, cosmological parameters from LSS, superclusters

ArXiv ePrint: 1109.4126 


\section{Contents}

1 Introduction $\quad 1$

2 The ISW effect $\quad 3$

3 Expected signal from superstructures in $\Lambda$ CDM 4

3.1 The number density of structures on different scales 5

3.2 Mean radial profiles 5

$\begin{array}{lll}3.3 & \text { Temperature signal } & 7\end{array}$

4 Comparing theory to observation $\quad 8$

4.1 The measured ISW signal of superstructures 8

4.2 Comparison to the theoretical expectation 9

$\begin{array}{ll}\text { 4.2.1 Accounting for selection effects } & 10\end{array}$

$\begin{array}{lll}\text { 4.2.2 Potential systematic errors } & 12\end{array}$

$\begin{array}{lll}4.3 & \text { Comparison with earlier results } & 14\end{array}$

5 Summary and prospectives $\quad 15$

6 Acknowledgements 17

\section{Introduction}

The standard 'concordance' $\Lambda \mathrm{CDM}$ cosmological model fits many different observations, including the luminosity distance-redshift relation of Type Ia supernovae (SNe Ia) (e.g. [1-3]), the anisotropies in the CMB [4], the locally measured Hubble parameter (e.g. [5]) and baryon acoustic oscillations (e.g. [6] — but see $[7,8]$ ). These observations, when interpreted assuming the homogeneous and isotropic Friedman-Robertson-Walker metric, imply that the expansion of the universe is accelerating, from which it is inferred that the universe is presently dominated by a cosmological constant ('dark energy') with negative pressure. It is important to note that this evidence is mostly geometrical, being based on interpreting measurements of distances - made using 'standard rulers' (the sound horizon at last scattering) and 'standard candles' (SNe Ia) - as due to accelerated expansion. The same data can equally be fitted without dark energy if, e.g., the isotropic but radially inhomogeneous Lemaïtre-Tolman-Bondi metric is assumed and other assumptions such as a power-law spectrum for the primordial density perturbations are relaxed (e.g. $[9,10])$.

Given that dark energy is a complete mystery from a physical viewpoint, it is therefore imperative to examine the observational evidence for its dynamical effects. For example, the decay of gravitational potentials after dark energy begins to dominate (at redshift $z \lesssim 1$ ) should lead to secondary CMB anisotropies as the CMB photons traverse regions of over- or under-density - the ISW effect [11]. If the universe is spatially flat, then detection of the ISW effect through cross-correlation of the CMB with large-scale structure would provide 
direct evidence of dark energy's negative pressure, hence crucial confirmation of the $\Lambda$ CDM model $[12] .^{1}$

To detect the ISW effect with $5 \sigma$ significance in CMB-galaxy cross-correlations requires $z$ measurements for over 10 million galaxies $[14,15]$. Such datasets are not yet available but several authors (e.g., [16-23]) have examined smaller source catalogues and reported detections with $<3 \sigma$ significance; however, [24] provides a skeptical view of some of these analyses. Conversely, some authors $[25,26]$ were unable to reject the null hypothesis (no ISW effect) and others [27] even found a slight anti-correlation rejecting $\Lambda$ CDM at $2-$ $3 \sigma$ significance. Some groups have combined different data sets to increase the detection significance above $4 \sigma[28,29]$ but it has been argued that these analyses have underestimated the error bars [30].

Much of the uncertainty in full-sky studies arises from the difficulty in reconstructing the underlying density field from galaxy survey data, given Poisson noise in the galaxy distribution. A different approach to this problem is followed by Granett et al. [31, 32], who study the Sloan Digital Sky Survey (SDSS) Data Release 6 (DR6) luminous red galaxies (LRGs). They use 3D galaxy information rather than the projected 2D density, and select only the most extreme density perturbations, which are unambiguously identified despite Poisson noise. Along the lines of sight corresponding to these 'superstructures' they report a $4.4 \sigma$ detection of the ISW effect, which is the most significant reported detection to date. This approach also provides information about the sizes and distribution of extreme structures in the universe so can be used to check the consistency of the standard $\Lambda$ CDM model of structure formation, in particular whether the primordial fluctuations were indeed gaussian.

However the magnitude of the temperature signal reported in Ref. [31] is surprisingly large and has been argued $[33,34]$ to be quite inconsistent with $\Lambda$ CDM. In response, it has been noted [35] that the assumed profile of the superstructures has a big effect on the signal, and that the 'compensated top-hat' profile adopted in Refs. [33, 34] is not the most appropriate. Using an alternative profile that is calibrated against $N$-body simulations and adopting a template-fit approach, it is claimed [36] that the discrepancy with $\Lambda \mathrm{CDM}$ is only at the $2 \sigma$ level.

Our aim in this paper is to clarify this important issue. We calculate the expected temperature signal from these superstructures making no a priori assumptions about their nature except that they arose in a $\Lambda$ CDM cosmology with gaussian primordial density perturbations. Our analysis uses linear perturbation theory, but non-linear corrections are argued to be sub-dominant at the relevant length scales and redshift. We use a statistical treatment of (initially) gaussian perturbations [37] to calculate the expected number densities and the expected profiles of the superstructures. These profiles are not significantly different from those used in Ref. [35], and we do find a factor of $\sim 3$ increase in the ISW signal over that calculated earlier [33, 34]. However, contrary to Ref. [36] we find that the expected value of the signal is still discrepant at $>3 \sigma$ with the observations reported by G08a. We demonstrate that this difference in the conclusions arises due to the interpretation of the template-fitting approach used in Ref. [36] and argue that a correct interpretation would lead to conclusions compatible with those presented here.

In Section 2 we briefly review the ISW effect and in Section 3 we calculate the expected temperature signal of superstructures in the standard $\Lambda$ CDM model. In Section 4.1 we

\footnotetext{
${ }^{1}$ The ISW effect should also boost low multipoles in the CMB angular power spectrum, whereas these are in fact anomalously low on the observed sky. However given the large cosmic variance on these scales and galactic foreground systematics, this discrepancy is not thought to be significant [13].
} 
describe the key features of the observational strategy [31,32] which must be accounted for before making a comparison with the theoretical calculation. In Section 4.2 we show that even if Refs. [31, 32] had selectively picked out the regions in the survey with the biggest ISW signal, there is still a significant discrepancy with the $\Lambda$ CDM expectation. In Section 4.3 we discuss the reasons for the difference between our result and that of Ref. [36].

Finally in Section 5 we turn to possible alternative explanations outside the standard cosmological model. Modifications to the growth rate of perturbations from the $\Lambda$ CDM prediction could be responsible for the discrepancy, but any alternative model must also match the other observational successes of $\Lambda \mathrm{CDM}$. A plausible explanation is that the effect is due to non-gaussianity of the primordial perturbations, but further work is needed to calculate its effect on the expected signal. Until this discrepancy is clarified, the observations reported in Ref. [31] do not appear to be compatible with the ISW effect expected in the standard cosmology.

\section{The ISW effect}

In a universe with matter density $\Omega_{\mathrm{m}}=1$ and no dark energy, density perturbations $\delta$ grow at exactly the same rate as the scale factor of the universe $a\left(\equiv(1+z)^{-1}\right)$, so at the linear level there is no evolution of the gravitational potential $\Phi(\propto-\delta / a)$. However, in a $\Lambda$ CDM universe, $a$ grows faster than (linear) density perturbations, so perturbations in $\Phi$ decay with time. For a CMB photon passing through an overdense region the energy gained while falling in is not cancelled by the energy lost in climbing out of the evolved, shallower, potential well. Overdense regions (clusters) therefore appear as hot spots in the CMB; conversely, underdense regions (voids) will appear as cold spots as the photon loses more energy climbing the potential hill than it gains subsequently while descending.

The temperature fluctuation $\Delta T(\hat{n})$ induced along direction $\hat{n}$ is [11]:

$$
\Delta T(\hat{n})=\frac{2}{c^{3}} \bar{T}_{0} \int_{0}^{r_{\mathrm{L}}} \dot{\Phi}(r, z, \hat{n}) a \mathrm{~d} r,
$$

where $\bar{T}_{0}$ is the mean CMB temperature, $r_{\mathrm{L}}$ is the radial comoving distance to the last scattering surface (LSS), $\dot{\Phi}(r, z, \hat{n})$ is the time derivative of the gravitational potential along the photon geodesic and $c$ is the speed of light.

The Poisson equation relates $\Phi$ to the density contrast $\delta \equiv(\rho-\bar{\rho}) / \bar{\rho}$ (where $\bar{\rho}(t)$ is the mean density) through:

$$
\nabla^{2} \Phi(\mathbf{x}, t)=4 \pi G \bar{\rho}(t) a^{2} \delta(\mathbf{x}, t) .
$$

This can be written in Fourier space as

$$
\Phi(\mathbf{k}, t)=-\frac{3}{2}\left(\frac{H_{0}}{k}\right)^{2} \Omega_{\mathrm{m}} \frac{\delta(\mathbf{k}, t)}{a},
$$

where $H_{0}$ is the current Hubble parameter. Taking the time derivative of this equation yields:

$$
\dot{\Phi}(\mathbf{k}, t)=\frac{3}{2}\left(\frac{H_{0}}{k}\right)^{2} \Omega_{\mathrm{m}}\left[\frac{\dot{a}}{a^{2}} \delta(\mathbf{k}, t)-\frac{\dot{\delta}(\mathbf{k}, t)}{a}\right] .
$$

We assume that linear theory holds on the large scales of interest hence perturbations grow as $\delta(\mathbf{k}, t)=D(t) \delta(\mathbf{k}, z=0)$, where $D(t)$ is the linear growth factor. A numerical 
simulation has shown that non-linear effects represent only a $10 \%$ correction at the low redshifts we are interested [38]. ${ }^{2}$ In this approximation,

$$
\dot{\Phi}(\mathbf{k}, z)=\frac{3}{2}\left(\frac{H_{0}}{k}\right)^{2} \Omega_{\mathrm{m}} \frac{H(z)}{a}[1-\beta(z)] \delta(\mathbf{k}, z),
$$

where $\beta(z) \equiv \mathrm{d} \ln D / \mathrm{d} \ln a$ is the linear growth rate. Hence the time evolution is captured by the ISW linear growth factor, $G(z)=H(z)(1-\beta(z)) D(z) / a$. For an $\Omega_{\mathrm{m}}=1$ universe, $\beta(z)=1$ for all $z$ so there is no ISW effect.

Given the density profile $\delta$ of any isolated superstructure, Eqs. (2.1) and (2.5) can be used to calculate the temperature fluctuation it induces in the CMB. Assuming spherical symmetry of the density profile, Eq. (2.5) in real-space becomes:

$$
\dot{\Phi}(r, z)=\frac{3}{2} \Omega_{\mathrm{m}} H_{0}^{2} G(z) F(r),
$$

where

$$
F(r)=\int_{0}^{r} \frac{r^{\prime 2}}{r} \delta\left(r^{\prime}\right) \mathrm{d} r^{\prime}+\int_{r}^{\infty} r^{\prime} \delta\left(r^{\prime}\right) \mathrm{d} r^{\prime},
$$

with $\delta\left(r^{\prime}\right)$ evaluated at redshift $z=0$. Thus $F(r)$ contains all information about the structure in question, while the assumed cosmology enters through the prefactor and the ISW growth factor $G(z)$ in Eq. (2.6).

\section{Expected signal from superstructures in $\Lambda \mathrm{CDM}$}

'Superstructures' refer to density perturbations extending over $\gtrsim 100 h^{-1} \mathrm{Mpc}$ and should not be thought of as non-linear collapsed structures in the usual sense, rather as smooth hills and valleys in the density distribution. Collapsed structures form only where the density perturbation $\delta(\mathbf{r})$ exceeds unity, which happens on much smaller scales than those of interest here.

It is stated in Ref. [31] that the most extreme structure in the $\left(500 h^{-1} \mathrm{Mpc}\right)^{3}$ box of the Millennium $N$-body simulation [39], when placed at $z=0$, would produce a signal of $\Delta T \sim 4.2 \mu \mathrm{K}$. However when the signal distribution for supervoids with the densities and sizes reported in Ref. [32] was calculated, the answer was only $\langle\Delta T\rangle=-0.42 \mu \mathrm{K}$ [33]. These authors [33] assumed a 'compensated top-hat' density profile motivated by the asymptotic final state of a void [40]. A similar profile was assumed by other authors [34] who found a similar average signal $\langle\Delta T\rangle=-0.51 \mu \mathrm{K}$ for the 50 most extreme density perturbations of fixed radius $r=130 h^{-1} \mathrm{Mpc}$ expected in a $\Lambda \mathrm{CDM}$ cosmology. Subsequently it was argued $[35,36]$ that this profile is not the appropriate choice for density perturbations on $\gtrsim 100 \mathrm{~h}^{-1} \mathrm{Mpc}$ scales; instead an uncompensated density profile was motivated from gaussian statistics, and was found [35] to give larger values of $\Delta T$. Using this profile Ref. [36] claimed only a $2 \sigma$ discrepancy between the $\Lambda \mathrm{CDM}$ prediction and observation. However, as we discuss in Section 4.3, the interpretation of [36] implies underdense regions with a physical density contrast $\delta<-1$, which is physically impossible.

It is thus necessary to revisit this issue. Using the statistics of a homogeneous, isotropic, gaussian density field, we now derive the expected mean density profiles of superstructures

\footnotetext{
${ }^{2}$ Both the linear and non-linear effects grow with time, however at late times and large scales $(\sim$ $100 h^{-1} \mathrm{Mpc}$ ) the linear effect dominates while at early times (when $\Omega_{\Lambda} \simeq 0$ ), both effects are smaller but the non-linear effect dominates.
} 
of all density contrasts and all sizes, as well as the expected number density of such superstructures.

\subsection{The number density of structures on different scales}

We identify superstructures of different sizes with extrema of the linear density perturbation field $\delta(\mathbf{r})$ when smoothed over different scales. Overdensities correspond to peaks of the smoothed field and underdensities to troughs. In the $\Lambda \mathrm{CDM}$ model, $\delta(\mathbf{r})$ is a homogeneous and isotropic, gaussian-distributed random field and the statistical properties of the maxima and minima have been calculated in Ref. [37]. We briefly review below their key results and introduce necessary notation.

Let $P(k, t)$ denote the matter power spectrum, defined as the Fourier transform of the 2-point correlation function $\xi(r, t)$ of the density field at time $t$. Define a set of spectral moments weighted by powers of $k$ :

$$
\sigma_{j}^{2}(t)=\int \frac{k^{2} \mathrm{~d} k}{2 \pi^{2}} W^{2}\left(k R_{\mathrm{f}}\right) P(k, t) k^{2 j},
$$

where $W\left(k R_{\mathrm{f}}\right)$ is the window function appropriate to the filter used to smooth the density field, and $R_{\mathrm{f}}$ is the (comoving) smoothing scale. Thus $\sigma_{0}$ is just the standard rms fluctuation of the smoothed density field. Using a gaussian filter, $W\left(k R_{\mathrm{f}}\right)=\exp \left(-k^{2} R_{\mathrm{f}}^{2} / 2\right)$, we define the parameters:

$$
\gamma \equiv \frac{\sigma_{1}^{2}}{\sigma_{2} \sigma_{0}}, R_{*} \equiv \sqrt{3} \frac{\sigma_{1}}{\sigma_{2}} .
$$

The (comoving) differential number density $\mathcal{N}_{\max }(\nu)$ of maxima of height $\delta_{0}=\nu \sigma_{0}$ is then [37]:

$$
\mathcal{N}_{\max }(\nu) \mathrm{d} \nu=\frac{1}{(2 \pi)^{2} R_{*}^{3}} \mathrm{e}^{-\nu^{2} / 2} G(\gamma, \gamma \nu) \mathrm{d} \nu .
$$

The function $G(\gamma, \gamma \nu)$ is given by Eq. (A19) of Ref. [37]; we use a fitting form, accurate to better than 1\%, given in their Eqs. (4.4) and (4.5). The density of minima is related simply to that of maxima through: $\mathcal{N}_{\min }(\nu)=\mathcal{N}_{\max }(-\nu)$.

\subsection{Mean radial profiles}

Having identified superstructures with the maxima or minima in the smoothed density field, we wish to determine the mean radial variation of the density field in the neighbourhood of these extrema. Ref. [37] shows that, given a maximum $\delta=\delta_{0}$ at $\mathbf{r}=0$, the mean shape in the vicinity of this point after averaging over all possible orientations of the principal axes as well as all values of the curvature at $\mathbf{r}=0$ is:

$$
\bar{\delta}(r)=\frac{\delta_{0}}{\left(1-\gamma^{2}\right)}\left(\psi+\frac{R_{*}^{2}}{3} \nabla^{2} \psi\right)-\frac{\left\langle x \mid \delta_{0}\right\rangle \sigma_{0}}{\gamma\left(1-\gamma^{2}\right)}\left(\gamma^{2} \psi+\frac{R_{*}^{2}}{3} \nabla^{2} \psi\right),
$$

where $\psi(r) \equiv \xi(r) / \xi(0)$ is the normalised density-density correlation function, and $x=$ $-\nabla^{2} \delta / \sigma_{2}$. The expectation value of $x$ given a peak of height $\delta_{0}$ at $\mathbf{r}=0$ is approximately

$$
\left\langle x \mid \delta_{0}\right\rangle=\gamma \nu+\Theta(\gamma, \gamma \nu),
$$

where $\nu=\delta_{0} / \sigma_{0}$, and $\Theta(\gamma, \gamma \nu)$ is given by the fitting function (6.14) of Ref. [37]. It follows that Eq. (3.4) can be rewritten [41] as:

$$
\bar{\delta}(r, t)=\frac{1}{\sigma_{0}} \int_{0}^{\infty} \frac{k^{2}}{2 \pi^{2}} \frac{\sin (k r)}{k r} W^{2}\left(k R_{\mathrm{f}}\right) P(k, t)\left[\frac{\nu-\gamma^{2} \nu-\gamma \Theta}{1-\gamma^{2}}+\frac{\Theta R_{*}^{2} k^{2}}{3 \gamma\left(1-\gamma^{2}\right)}\right] \mathrm{d} k,
$$



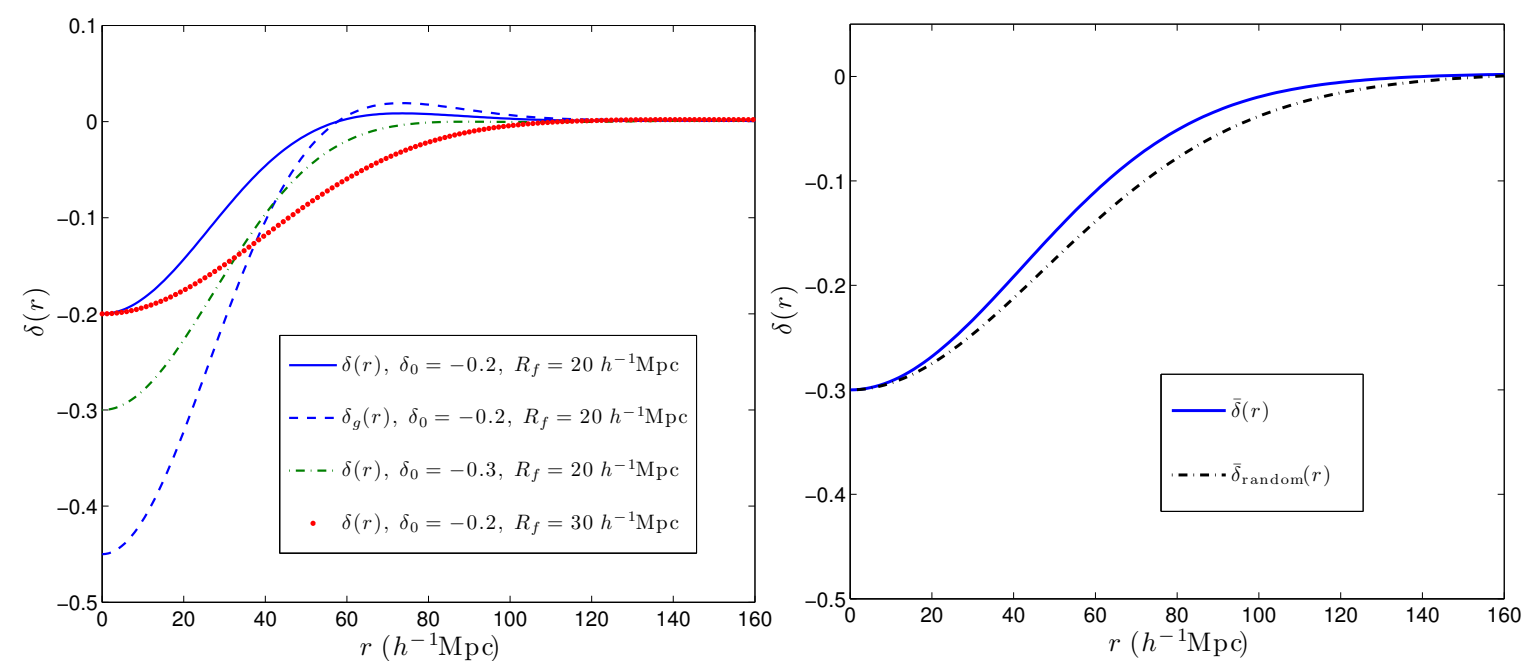

Figure 1. Left panel: Mean radial profiles for voids obtained from Eq. (3.6) for different values of central underdensity $\delta_{0}$ and the smoothing scale $R_{\mathrm{f}}$. The blue (solid) line and the green (dash-dot) line are for the same $R_{\mathrm{f}}\left(=20 h^{-1} \mathrm{Mpc}\right)$ but different values of $\delta_{0}$, whereas the red (dotted) profile has the same $\delta_{0}(=-0.2)$ as the blue (solid) profiles, but a larger smoothing scale. The blue (dashed) curve is the biased galaxy density contrast corresponding to matter density contrast given by the blue (solid) line, with bias factor $b=2.25$ as is appropriate for LRGs. Right panel: Comparison between the two methods of calculating mean radial profiles. The black (dash-dot) line represents $\bar{\delta}_{\text {random }}(r)$ from Eq. (3.7) for $\delta_{0}=-0.3$ and $R_{\mathrm{f}}=30 h^{-1} \mathrm{Mpc}$. The blue (solid) line is the corrected profile $\bar{\delta}(r)$ from Eq. (3.6) for the same parameter values.

which we use for numerical evaluation of profiles. Note that $\gamma$ and $R_{*}$ depend on the smoothing scale $R_{\mathrm{f}}$.

We make the simplifying approximation that the average ISW signal for a large number of superstructures is the same as the signal due to a superstructure with the mean profile (3.6) - this is justified because of the linear relationship between $\Delta T, \Phi$ and $\delta$. The actual distribution of the size of structures depends on the distribution of $x$ values, which can be obtained from Eq. (7.5) of Ref. [37]. In the left panel of Fig. 1 we plot some underdense profiles for selected values of $\delta_{0}$ and $R_{\mathrm{f}}$ (these examples are chosen for clarity and are not representative of the most likely actual underdensities). Note that the size of the structures is much larger than the smoothing scale $R_{\mathrm{f}}$.

To identify superstructures in galaxy surveys (the methodology of Ref. [31] is discussed in more detail in Section 4.2) the galaxy density contrast $\delta_{g}$ is assumed to be linearly biased with respect to the matter density: $\delta_{g}=b \delta$. Denoting by $\rho_{\mathrm{sl}}$ the value of the density field at turnover, and by $\rho_{0}$ the minimum density at the centre, a selection cut is made on on $w \equiv \rho_{\mathrm{sl}} / \rho_{0}$, amounting to a lower bound on the absolute value of $\delta_{0}$. This avoids false detections of over- and under-dense regions that are just Poisson fluctuations in galaxy number counts and ensures that only extreme superstructures are included in the ensemble.

The definition of the radius $R_{\mathrm{v}}$ of any of the voids shown in Fig. 1 is slightly ambiguous. We choose it to be the radius of turnover in the density profile less $R_{\mathrm{f}}$, since smoothing necessarily increases the radius somewhat. This is a small correction since in general $R_{\mathrm{v}} \gg$ $R_{\mathrm{f}}$.

The mean profile about a random point with a density contrast $\delta_{0}$ (i.e., a point which 
is not necessarily an extremum of the density field) is simply [42]

$$
\bar{\delta}_{\text {random }}(r)=\delta_{0} \psi(r) \text {. }
$$

This should be compared with Eq. (3.4), which is the appropriate mean profile about points that are also known to be extrema. Eq. (3.7) is the form of the density profile used in Refs. [35, 36]. In Ref. [36] this is matched to the mean density profile about randomly chosen points in $N$-body simulations and is found to be a good approximation, as expected. It must be emphasised that as we identify superstructures with points of extrema in the density field, consistency demands that we use the corrected form of Eqs. (3.4) and (3.6) rather than Eq. (3.7). This gives a profile that is slightly steeper than that used in $[35,36]$.

In practical terms, however, the choice of profile between Eq. (3.4) and Eq. (3.7) will have little effect on our conclusions as the difference between these profiles is small. In the right panel of Fig. 1 we show as an example the mean profiles $\bar{\delta}(r)$ and $\bar{\delta}_{\text {random }}$ for $\delta_{0}=-0.3$ and $R_{\mathrm{f}}=30 h^{-1} \mathrm{Mpc}$. It can be seen that the two are similar: in fact, for most values of $\delta_{0}$ and $R_{\mathrm{f}}$ and over almost the entire range of $r, \bar{\delta}_{\text {random }}(r)$ lies within the $\pm 1 \sigma$ dispersion around $\bar{\delta}(r)$ of the profiles of extrema (this is not shown here but some examples can be seen in Fig. 8 of Ref. [37]).

Of course the quantity determining $\Delta T$ in Eq. (2.1) is not $\delta(r)$ but an integral over $F(r)$, which is itself an integral over $\delta(r)$, and hence small differences may be magnified. Therefore, to explicitly check that the choice of profile is not responsible for the difference between our results and those of [36], we also repeat the calculation of the expected temperature signal described below using $\bar{\delta}_{\text {random }}(r)$ to model structures.

\subsection{Temperature signal}

The ISW signal of any individual superstructure will be too small compared to the primordial CMB anisotropies to be observable. Therefore, what is measured is the average temperature fluctuation along the lines of sight of a selected sample of either over- or under-densities. The primordial anisotropies are uncorrelated with the large scale structure and average out so given a large enough sample the correlated ISW signal eventually dominates. Our calculation of this averaged signal is done as follows.

At a given $z$, we use Eq. (3.6) to calculate the matter and galaxy density profiles about extrema of the density field as functions of $\delta_{0}$ and $R_{\mathrm{f}}$ and obtain $\dot{\Phi}$ along the line of sight as discussed in Section 2. This enables us to calculate $\Delta T\left(\theta ; \delta_{0}, R_{\mathrm{f}}\right)$ where $\theta=0^{\circ}$ is the line of sight passing through the centre of the superstructure. To compare with the observations we first apply the selection criterion on $\delta_{0}$ through the limit on $w$. Then, to calculate the expectation value $\langle\Delta T\rangle$ for the resulting ensemble, we weight the results appropriately with the number density (3.3) of extrema. Hence for an ensemble of voids:

$$
\langle\Delta T\rangle=\frac{\iint W(\theta) \Delta T\left(\theta ; \delta_{0}\right) \mathcal{N}_{\min } \sigma_{0}^{-1} \mathrm{~d}^{2} \theta d \delta_{0}}{\pi \theta_{\mathrm{c}}^{2} \int \mathcal{N}_{\min } \sigma_{0}^{-1} \mathrm{~d} \delta_{0}},
$$

where $0 \leq \theta \leq \theta_{\text {out }} ; W(\theta)$ is a filter of angular width $\theta_{\text {out }}$ chosen in order to match that used in the actual observation and $-1 \leq \delta_{0} \leq \delta_{0}^{\mathrm{c}}$, where $\delta_{0}^{\mathrm{c}}$ is the (mildly $R_{\mathrm{f}}$-dependent) cutoff on the minimum underdensity required to pass the selection criterion. The choice of $R_{\mathrm{f}}$ determines the mean radial size of the voids included in the ensemble; although structurefinding algorithms may not have an explicit size dependence, in practice there is obviously a lower limit on the size of the over- or under-density that can be reliably found. As smaller 
structures are overwhelmingly more probable than larger ones, it is important to capture this effect and we discuss this in more detail in the next Section. For a given ensemble of voids specified by $R_{\mathrm{f}}$ and $\delta_{0}^{\mathrm{c}}$, the observed temperature signals $\Delta T$ will be distributed about this mean value. By calculating $\left\langle\Delta T^{2}\right\rangle$ in a similar fashion to Eq. (3.8) we can estimate the standard deviation of this distribution simply as $\left(\left\langle\Delta T^{2}\right\rangle-\langle\Delta T\rangle^{2}\right)^{1 / 2}$.

The expected signal from an ensemble of clusters follows in an exactly analogous manner to Eq. (3.8).

\section{Comparing theory to observation}

\subsection{The measured ISW signal of superstructures}

To compare our expectation for the ISW signal to the measurement made by Ref. [31], it is necessary to follow the same selection procedure. They use a sample of 1.1 million LRGs in the range $0.4<z<0.75$ (median $z=0.52$ ) from the SDSS DR6 [43], which covers 7500 degree $^{2}$ on the sky and occupies a volume of $5 h^{-3} \mathrm{Gpc}^{3}$. They search for 'supervoids' and 'superclusters' using two publicly-available structure-finding algorithms: ZOBOV (ZOnes Bordering On Voidness; [44]) for supervoids, and VOBOZ (VOronoi BOund Zones; [45]) for superclusters.

It is necessary to mimic the way in which these algorithms select structures in choosing the ensemble for which to calculate $\langle\Delta T\rangle$ from Eq. (3.8). ZOBOV uses a parameter-free Voronoi tessellation to estimate the density at each galaxy in the sample, based on the distance to its nearest neighbours. Around each density minimum it then finds the region of the density depression or supervoid. (Of course large voids can contain multiple smaller voids, or even isolated high-density regions.) The 'significance' of the depression is estimated by comparing the density contrast, $w$ (defined as the ratio of the density at the lip of the void to the density at its minimum) to an uniform Poisson point sample. This yields the likelihood that a void of density contrast $w$ could arise from Poisson noise, i.e. that it is a false positive detection; a $3 \sigma$ cut is then applied on the likelihood which translates to requiring $w>w_{\mathrm{c}}=2.0$ on the density contrast [44]. This procedure yields 50 supervoids, the properties of which are tabulated in Ref. [32]. The VOBOZ supercluster finder uses the same algorithm but applied to the inverse of the density field, with density contrast defined as the ratio of the peak density to the density at the edge of the structure. However, now the $3 \sigma$ cut on the likelihood that an overdensity of given $w$ could have arisen due to Poisson noise corresponds to $w>6.8$ [45]. In fact the authors impose a tighter cut: $w>8.35$ in order to obtain exactly 50 such superclusters; their properties are also tabulated in Ref. [32].

Ref. [31] report a search for the ISW signals of these superstructures using an inversevariance weighted combination of the WMAP 5-year Q, V and W maps [46] with foreground subtracted and the KQ75 mask applied. They build stacked images by averaging the CMB temperature in the regions around the lines of sight passing through the centres of the identified superstructures and use a compensated top-hat filter of width $\theta_{\mathrm{c}}$ in order to perform the averaging. This corresponds to making the choice in Eq. (3.8):

$$
W(\theta)=\left\{\begin{array}{cc}
1, & 0 \leq \theta \leq \theta_{\mathrm{c}} \\
-1, & \theta_{\mathrm{c}}<\theta \leq \theta_{\text {out }}
\end{array}\right.
$$

with $\theta_{\text {out }}=\sqrt{2} \theta_{\mathrm{c}}$. For a filter radius $\theta_{\mathrm{c}}=4^{\circ},{ }^{3}$ the sample of supervoids gives $\langle\Delta T\rangle=$ $-11.3 \pm 3.1 \mu \mathrm{K}$, and the sample of superclusters $\langle\Delta T\rangle=7.9 \pm 3.1 \mu \mathrm{K}$. When averaged

\footnotetext{
${ }^{3}$ This choice is apparently motivated by the expectation that the CMB-galaxy cross-correlation should
} 
together (with the negative of the supervoid image added to the superclusters) this gives $\langle\Delta T\rangle=-9.6 \pm 2.2 \mu \mathrm{K}$, i.e. a $4.4 \sigma$ detection of a non-zero correlation.

The quoted error bars are determined by Monte Carlo simulations in two different ways: by stacking images of the actual CMB map in random directions, and by keeping the directions fixed but using model CMB maps. Note that this explicitly accounts for cosmic variance in the CMB fluctuations. The theoretical error bars described in Section 3.3 account for the distribution of voids of different depths and sizes. Misidentification of structures in the dark matter density field can also occur due to shot noise in the galaxy distribution. This means that the structures identified as the $N$ most extreme fluctuations will in fact be a random sample of $N$ structures drawn from among the $M$ most extreme, for some $M \geq N$. In Section 4.2.1, we make the optimistic assumption that $N=M$ such that the expected signal is maximised.

A caveat that should be noted, however, is that the choice in Ref. [31] of using exactly 50 supervoids and superclusters each is made to obtain the largest signal-to-noise ratio. For both $N=30$ and $N=70$ a somewhat lower significance detection is reported compared to $N=50$. As the distribution of $\Delta T$ values for the voids is very non-Gaussian, this choice of $N$ could be important. Fluctuations due to the underlying CMB anisotropy dominate at small $N$ and false positive identifications of structures increase at large $N$ so the signal-to-noise ratio is indeed expected to have a maximum at some intermediate $N$. However more detailed study is needed to quantify whether this is expected to be at $N \simeq 50$. In this paper we take the reported observations at face value and our interpretation is based on the data for $N=50$.

\subsection{Comparison to the theoretical expectation}

We assume for simplicity that all the superstructures are located at the mean redshift $z=0.52$ and adopt the standard $\Lambda$ CDM cosmological model with $\Omega_{\mathrm{m}}=0.29, \Omega_{\Lambda}=0.71, n_{\mathrm{s}}=0.96$ and $\sigma_{8}=0.83$ (with $h=0.69$ where required) to obtain the matter power spectrum at $z=0.52$ using CAMB [47]. ${ }^{4}$ The bias factor for LRGs is taken to be $b=2.25$. These parameters are kept fixed for the purposes of calculation, but as we discuss later, varying them within the bounds set by other observational data has no effect on the conclusions.

Our first finding is that there are no overdense superstructures within the linear regime (i.e. with $\delta_{0}<1$ ) which meet the VOBOZ $3 \sigma$-significance selection criterion that $w>6.82$. Such a ratio of densities (between the lip of an overdensity and its centre) can be achieved, but only for non-linear collapsed structures. We conclude that Table 5 of Ref. [32] does not list the most overdense large-scale linear perturbations, but the (mild) large-scale linear perturbations that happen to contain the most overdense small-scale collapsed structures. This means that the relative ranking and selection of large-scale linear overdensities made in Ref. [32] is done on the basis of the small-scale collapsed structures they contain. The statistical effect on the CMB temperature of small collapsed structures could be calculated, but we cannot model the criteria by which these overdense regions were selected for study. Note however that our calculations below for the maximum possible amplitude of ISW signal from superstructures holds equally well for over- and under-densities in the linear regime.

peak at about $4^{\circ}[22]$. Ref. [31] reports repeating the observation with a few other widths, $3^{\circ} \leq \theta_{\mathrm{c}} \leq 5^{\circ}$ and obtaining a maximum detection significance for $\theta_{\mathrm{c}}=4^{\circ}$.

${ }^{4}$ These are the mean parameter values obtained from a fit to WMAP 7-year [4] and SDSS DR7 [48] data using COSMOMC [49]. 
Any contamination of the regions selected by VOBOZ will only reduce the expected signal, although we are unable to estimate by how much.

On the other hand, this problem of modelling the selection of structures for study does not arise for voids. Hence we concentrate only on the sample of underdense regions (i.e. supervoids), for which $\langle\Delta T\rangle_{\mathrm{obs}}=-11.3 \pm 3.1 \mu \mathrm{K}$. To calculate the expected $\langle\Delta T\rangle$ using Eq. (3.8), we must first choose the smoothing scale $R_{\mathrm{f}}$ and determine the distribution of void radii $R_{\mathrm{v}}$ in the ensemble using Eq. (7.5) of Ref. [37]. For each $R_{\mathrm{f}}$ we calculate $R_{\mathrm{v}}^{\text {min }}$ such that $95 \%$ of all voids in the ensemble have radius $R_{\mathrm{v}}>R_{\mathrm{v}}^{\min }$, this being a convenient way to characterise the ensemble. For $R_{\mathrm{f}}=20 h^{-1} \mathrm{Mpc}$, we find $R_{\mathrm{v}}^{\min } \sim 70 h^{-1} \mathrm{Mpc}$, which is similar to the mean radius of the voids in Table 4 of Ref. [32]; $;^{5}$ at this scale, the number of such voids that should satisfy the selection criterion is $N_{\mathrm{v}} \sim 10^{4}$. This is to be compared with the $N_{\mathrm{v}}=50$ voids that are actually tabulated in Ref. [32]. For the larger ensemble, we find an expectation value $\langle\Delta T\rangle=-0.3 \pm 0.2 \mu \mathrm{K}$, i.e. consistent with zero.

However, since Granett et al. see only a tiny fraction of the total number of supervoids expected in the SDSS DR6 volume, it is unreasonable to assume that this represents a fair sample. Some strong selection effects must be in operation; while these have no effect on our ISW prediction for individual voids, they will affect the selection of structures in the LRG distribution and thus the ensemble expectation value. Selection effects that can enhance the expected signal are a bias towards larger and deeper regions, implying that Granett et al. did not randomly select 50 of the $\sim 10^{4}$ expected supervoids but chose some sample that is skewed towards regions with larger $\Delta T$ values. We show below that the expected signal from the 50 most extreme regions is indeed $\sim 5$ times larger than $-0.3 \mu \mathrm{K}$; nevertheless the discrepancy with the observation in Ref. [31] is still $>3 \sigma$. Therefore, irrespective of how the regions were selected, tension remains with the expectation in the standard $\Lambda$ CDM model.

\subsubsection{Accounting for selection effects}

We first consider the possibility that the void-finding algorithm ZOBOV is sensitive only to the largest (and least common) voids in the matter distribution which produce the biggest ISW temperature signals. The average density of LRGs in the SDSS DR6 sample is roughly 1 galaxy per $\left(15 h^{-1} \mathrm{Mpc}\right)^{3}$. In underdense regions LRGs will be even more sparsely distributed so ZOBOV will certainly be less able to identify smaller underdense structures, thus biasing the sample towards larger voids.

In order to model the effect of such a selection bias, we increase the value of $R_{\mathrm{f}}$ in Eq. (3.8); this is equivalent to including only the $N_{\mathrm{v}}$ largest voids with radius $R_{\mathrm{v}} \geq R_{\mathrm{v}}^{\min }$ in the ensemble from which $\langle\Delta T\rangle$ is calculated. In the left panel of Fig. 2 we plot the expectation $\langle\Delta T\rangle$ as a function of $R_{\mathrm{v}}^{\text {min }}$. The orange cross-hatched area shows the region that is within $3 \sigma$ of the observed value $\langle\Delta T\rangle_{\text {obs }}=-11.3 \pm 3.1 \mu \mathrm{K}$. The theoretical value of $\langle\Delta T\rangle$ becomes marginally consistent with the observed value when $R_{\mathrm{v}}^{\min } \sim 170 \mathrm{~h}^{-1} \mathrm{Mpc}$. However the probability that within the SDSS survey volume there are 50 supervoids of radius $R_{\mathrm{v}} \geq 170 h^{-1} \mathrm{Mpc}$ which also meet the ZOBOV selection criterion is vanishingly small.

It is interesting to note that the 50 largest supervoids expected within the SDSS survey volume have $R_{\mathrm{v}}^{\min } \gtrsim 120 h^{-1} \mathrm{Mpc}$. The largest void radius reported in Ref. [32] is $125 h^{-1} \mathrm{Mpc}$ and the mean is $70 h^{-1} \mathrm{Mpc}$. We have argued that these values somewhat underestimate the size of the supervoids compared to our definition of $R_{\mathrm{v}}$, yet it seems unlikely that the difference could be so large that all the tabulated voids should have $R_{\mathrm{v}} \gtrsim 120 \mathrm{~h}^{-1} \mathrm{Mpc}$.

\footnotetext{
${ }^{5}$ At the radii reported here, the density has not yet reached the background level so the quoted values must be underestimates of the void size relative to our criterion.
} 

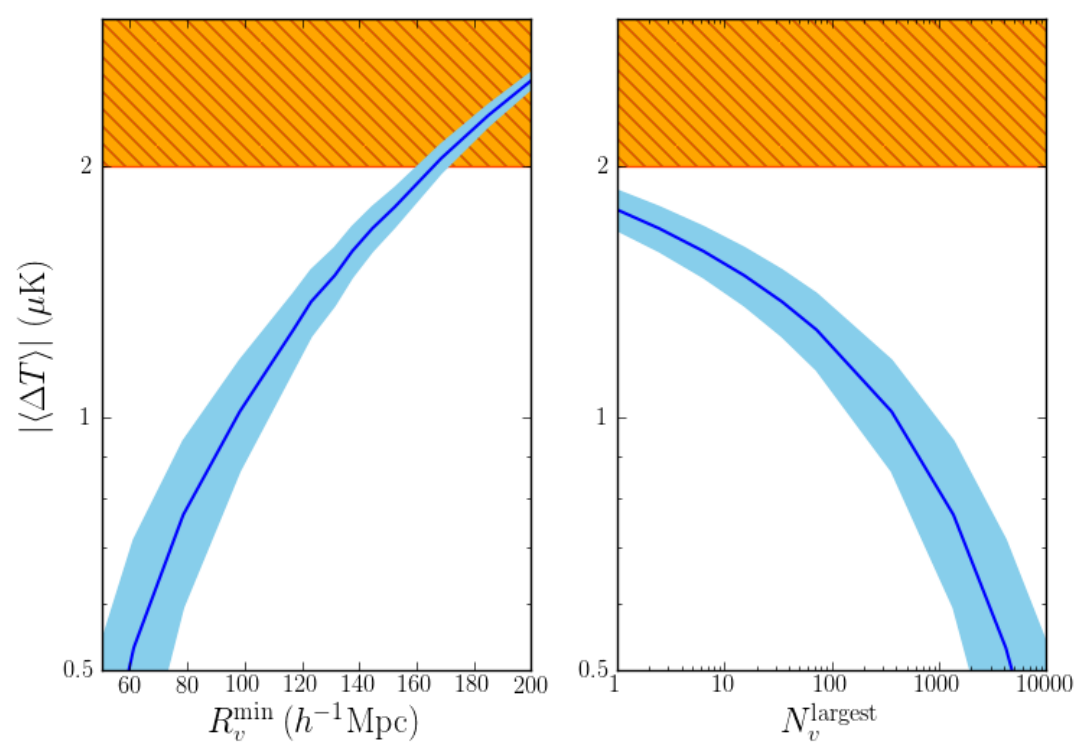

Figure 2. Left panel: The absolute value of $\langle\Delta T\rangle$ for an ensemble of voids which satisfy the ZOBOV selection condition on density (see text), as a function of the minimum radius of voids in the ensemble. The solid (blue) curve shows the mean value and the shaded (lighter blue) contours the $1 \sigma$ region. The (orange) cross-hatched area is the lower end of the $3 \sigma$ range of the observed value $\langle\Delta T\rangle_{\mathrm{obs}}=-11.3 \pm 3.1 \mu \mathrm{K}$. Right panel: As above, but showing $\langle\Delta T\rangle$ as a function of the number of voids in the ensemble from which the observed sample of 50 voids is to be drawn, when only the $N_{\mathrm{v}}$ largest voids also meeting the ZOBOV selection condition on density are included.

In the right-hand panel of Fig. 2 we plot $\langle\Delta T\rangle$ as a function of the size $N_{\mathrm{v}}$ of the ensemble of the largest supervoids that should exist within the SDSS volume. It is from this ensemble that the 50 observed supervoids should be regarded as having been drawn. It can be seen that even under the assumption that the VOBOZ algorithm selected exactly the 50 largest voids in the entire SDSS survey volume, the expected signal is only $\langle\Delta T\rangle=-1.33 \pm 0.13 \mu \mathrm{K}$ which is still discrepant by $>3 \sigma$ with the observed value. We conclude that the observed signal cannot be explained due to a simple bias towards selecting only the largest voids.

We repeated the calculation above using Eq. (3.7) to model the density profiles of the voids, as in Ref. [36], and obtained $\langle\Delta T\rangle=-1.58 \pm 0.12 \mu \mathrm{K}$ for the 50 largest voids. Despite the small increase compared to the value quoted above, this is still discrepant with observation at the same $>3 \sigma$ level. This conclusively demonstrates that, as anticipated, the difference between our conclusion and that of Ref. [36] is not due to the small correction included in $\bar{\delta}(r)$. Henceforth we use the corrected profile only.

We consider next whether the ZOBOV algorithm is more sensitive to deeper voids. In Table 4 of Ref. [32], the edge of most of the supervoids is defined at a radius where the density contrast is still negative. This means ZOBOV systematically underestimates the value of $w$ relative to our definition (where $\delta_{\text {edge }} \simeq 0$ ), so Granett et al. effectively used a more stringent cut on $w$ than we have done. We can model this effect by varying $\delta_{0}^{c}$ from the value determined by the stated algorithm. In the left-hand panel of Fig. 3 we plot as examples $\langle\Delta T\rangle$ as a function of $\delta_{0}^{\mathrm{c}}$ for $R_{\mathrm{v}}^{\min } \simeq 70 h^{-1} \mathrm{Mpc}$ (the mean radius of the supervoids in [31]) and $R_{\mathrm{v}}^{\min } \simeq 100 h^{-1} \mathrm{Mpc}$. The right-hand panel shows $\langle\Delta T\rangle$ as a function of $N_{\mathrm{v}}$, 

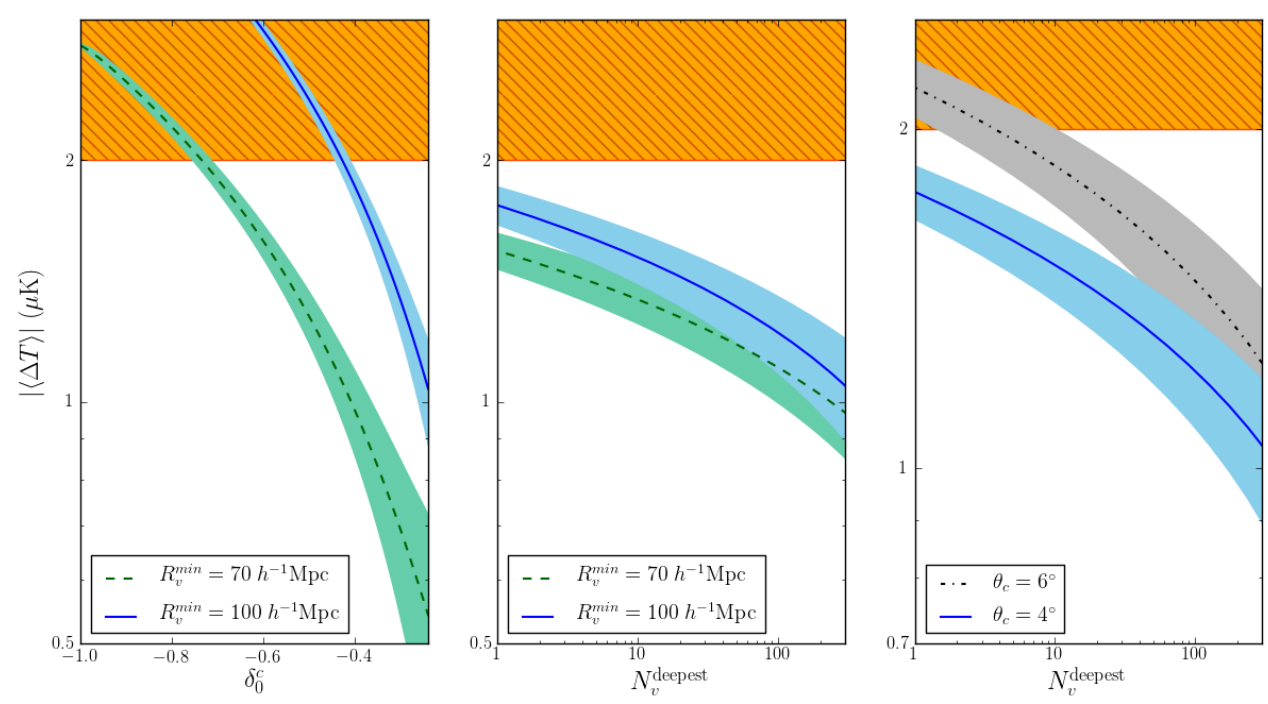

Figure 3. Left panel: The absolute value of $\langle\Delta T\rangle$ for an ensemble of supervoids which satisfy $\delta_{0}<\delta_{0}^{\mathrm{c}}$, as a function of $\delta_{0}^{\mathrm{c}}$. The dashed (green) curve shows the case when $R_{\mathrm{f}}$ is chosen such that voids with $R_{\mathrm{v}} \gtrsim 70 h^{-1} \mathrm{Mpc}$ are included in the ensemble; the solid (blue) curve is for $R_{\mathrm{v}} \gtrsim 100 h^{-1} \mathrm{Mpc}$. Shaded contours show the $1 \sigma$ region about the mean and the orange cross-hatched area is as in Fig. 2. Middle panel: As before, but showing $\langle\Delta T\rangle$ as a function of the number of voids in the ensemble from which the observed sample of 50 supervoids is to be drawn, when only the $N_{\mathrm{v}}$ deepest supervoids are included. Right panel: As before, but for two different choices of the radius $\theta_{c}$ of the compensating top-hat filter used in Eq. (3.8). The solid (blue) curve is the mean value for $\theta_{c}=4^{\circ}$, and the dot-dashed (black) curve for $\theta_{\mathrm{c}}=6^{\circ}$. Shaded regions show the $1 \sigma$ deviations from the mean.

the number of voids included in the ensemble when $\delta_{0}^{\mathrm{c}}$ is varied in each case. For the smaller radius, the ensemble is dominated by small voids with a small ISW effect, so increasing $R_{\mathrm{v}}^{\min }$ increases $\langle\Delta T\rangle$ at any $\delta_{0}^{\mathrm{c}}$. With $R_{\mathrm{v}}^{\min } \sim 100 h^{-1} \mathrm{Mpc},\langle\Delta T\rangle$ becomes marginally consistent with the observation for $\delta_{0}^{\mathrm{c}} \lesssim-0.5$. However, the probability of obtaining 50 supervoids with $R_{\mathrm{v}} \geq 100 h^{-1} \mathrm{Mpc}$ and $\delta_{0} \leq-0.5$ is negligibly small, as the right-hand panel clearly demonstrates. Therefore we conclude that in the standard cosmological picture there do not exist 50 isolated matter density voids in the SDSS volume that, irrespective of the method of selecting them, produce an average $\langle\Delta T\rangle$ compatible with observation. Our analysis, based on very conservative assumptions, shows that the claim [36] that the discrepancy between observation and the $\Lambda \mathrm{CDM}$ prediction is only $\sim 2 \sigma$ is an underestimate.

\subsubsection{Potential systematic errors}

We now discuss the expected corrections due to the simplifying assumptions we made in calculating the signal. We first consider the effect of varying the cosmological parameters from the mean values adopted in 4.2. This can affect the calculation of $\langle\Delta T\rangle$ in three ways: ${ }^{6}$ by altering the mean radial profile of the voids; by altering the expected number density of voids through Eq. (3.3); and by altering the ISW growth factor $G(z)$ and the integral

\footnotetext{
${ }^{6}$ Varying the bias factor only affects the detection of matter density voids via the observation of galaxies. By varying $\delta_{0}^{\mathrm{c}}$ as above we have already made the conservative assumption that only the most extreme matter voids that exist are detected. Therefore the bias factor can have no further effect on our conclusions.
} 
Eq. (2.1). The first two of these are dependent, through the moments $\sigma_{i}$, on the matter power spectrum $P(k)$. An increase in power increases the number density of points of extrema, and steepens the mean profile about these points. However, it also increases $\sigma_{8}$, which is already reasonably well constrained by WMAP [4] and SDSS DR7 [48] data. Within any reasonable range of $\sigma_{8}$, the change to the mean profile $\bar{\delta}(r)$ is completely negligible. Allowing $\sigma_{8}=0.87$, which is the $95 \%$ C.L. bound from WMAP + SDSS, does boost the relative number density of the largest voids by a factor of $\sim 1.5$. However, given the almost logarithmic dependence of $\langle\Delta T\rangle$ on $N_{\mathrm{v}}^{\text {largest }}$ evident from Fig. 2, the effect on $\langle\Delta T\rangle$ remains well within the statistical error bars already quoted.

The only remaining way for the cosmological parameters to affect $\langle\Delta T\rangle$ is through the value of $\Omega_{\mathrm{m}}$. Assuming a spatially flat model, this determines the growth factor $G(z)$, the constant pre-factor in $\dot{\Phi}$ and the photon geodesics (i.e. the relationship between $r$ and $z$ in Eq. (2.1)). Note that a smaller $\Omega_{\mathrm{m}}$ boosts $G(z)$ but decreases the pre-factor. $\Omega_{\mathrm{m}}$ is of course also constrained by other data, and we find that any value in the range $\Omega_{\mathrm{m}} \in(0.25,0.32)$ results in changes to $\langle\Delta T\rangle$ that are significantly smaller than the error bars already quoted. We conclude that the freedom to vary the $\Lambda \mathrm{CDM}$ model parameters is not enough to overcome the discrepancy with observation.

We have neglected non-linear effects and the time evolution of the void density profile $[40,50]$ in the calculation of $\langle\Delta T\rangle$. On small scales voids evolve non-linearly towards a compensated top-hat profile. These non-linear effects increase the depth of the cold spot at its centre but also produce a hot filamentary shell [38]. When averaged over angle $\theta$ it is not clear whether the net effect will lead to a net increase or decrease in $\langle\Delta T\rangle$. In fact, highly non-linear void profiles are known to produce a smaller ISW signal due to the effect of the overdense ridge at the boundary [34,35], so by using the linear theory profile we may be overestimating the expected signal. It must be stressed though that at small redshifts $z \sim 0.5$ and on the large scales of interest $\gtrsim 100 h^{-1} \mathrm{Mpc}$, the effects of non-linear evolution will in any case represent only a subdominant correction [38] and can justifiably be neglected here.

A more subtle assumption is that the real supervoid profiles are adequately captured by our smoothing prescription. The effect of the smoothing is to slightly broaden the $\delta(r)$ profile which in turn leads to broadening of the $\Delta T(\theta)$ profile. This may become a problem if the $\Delta T(\theta)$ profile is significantly broadened so the compensated top-hat filter of radius $4^{\circ}$ in Eq. (3.8) then underestimates the real signal. If this were the case then it would be more appropriate to use a broader top-hat. To check this, we repeated our analysis with a filter of radius $6^{\circ}$, which is a generous overestimate of the degree of broadening, and in the right panel of Fig. 3 we show the effect for the largest and deepest supervoids. As expected, increasing the filter radius does increase $\langle\Delta T\rangle$, but the effect is small. Even with a $6^{\circ}$ filter the expected signal remains $>3 \sigma$ discrepant with observations for $N_{\mathrm{v}}=50$; also as mentioned above, the actual effect on $\langle\Delta T\rangle$ due to the smoothing will be less than this extreme model. This indicates that our conclusion of a discrepancy will be independent of the details of the smoothing filter used.

We have made the simplifying assumption that the centres of all the superstructures lie at the same redshift $z=0.52$, which is the median redshift of the DR6 survey. This means we have not accounted for the possibility that two voids may lie at different redshift along roughly the same line of sight, thus increasing the temperature shift. Equally we do not account for the possibility that a void may lie in front of or behind an overdensity, thus decreasing the temperature shift. We expect that these two simplifications largely negate 
each other.

As a test of the robustness of our calculation, we compare our results with Fig. 1 of Ref. [38] which shows the ISW map from a cosmological $N$-body simulation for a volume comparable to the SDSS DR6, but at $z=0$ rather than $z=0.52$. The very largest density perturbations in this map yields a maximum ISW signal of $|\Delta T| \sim 4 \mu \mathrm{K}$ before applying a filter analysis. Taking into account that $\Delta T$ is more pronounced at smaller redshift, this tallies very well with our prediction from Fig. 3 that the most extreme supervoid in the SDSS volume should produce $\langle\Delta T\rangle \sim-2 \mu \mathrm{K}$.

As seen in the full-sky maps in Ref. [38], there are lines of sight along which the chance alignment of several large structures between us and the LSS several Gpc away can lead to isolated ISW 'cold spots' with $\Delta T<-10 \mu \mathrm{K}$. However, this is a cumulative effect of several structures over Gpc scales and does not affect our conclusions about the correlation between these cold spots and isolated structures at $z \sim 0.5$. (Note also that in the simulation the temperature profiles along such lines of sight are much broader that $4^{\circ}$ and so when averaged using a $4^{\circ}$ compensated top-hat filter would still give only a small $|\langle\Delta T\rangle|$.) Similarly, there will be several $4^{\circ}$ circles on the sky for which the underlying CMB anisotropy alone can give an average $\Delta T$ of similar magnitude - it is just such fluctuations that generate the observational uncertainty of $\pm 3.1 \mu \mathrm{K}$ of Ref. [31]. Such fluctuations are not due to any ISW effect and there is no reason why they should be correlated with large structures in the SDSS catalogue. Therefore, assuming only that the lines of sight were not chosen a posteriori, the observed correlation between galaxy structures and the CMB can only result from rare $(>3 \sigma)$ fluctuations or anomalously large density perturbations at $z \sim 0.5$.

Ref. [31] also reports the variation of the signal-to-noise ratio with the width of the compensating top-hat filter and finds a maximum at $\theta_{\mathrm{c}}=4^{\circ}$ for the combined sample of over-and under-dense structures. By contrast, for supervoids we find that $|\langle\Delta T\rangle|$ increases as $\theta_{\mathrm{c}}$ is raised from $4^{\circ}$ to $6^{\circ}$ (Fig. 3) and to larger angles. This is incompatible with a peak in the signal-to-noise ratio at $4^{\circ}$ and is another respect in which the observation of Ref. [31] cannot be modelled by linear structures in $\Lambda \mathrm{CDM}$.

\subsection{Comparison with earlier results}

We have provided a theoretical calculation of the expected average temperature signal in the CMB from superstructures observed in the SDSS LRG catalogue assuming a $\Lambda$ CDM cosmology with gaussian primordial density perturbations. On comparing this expectation to the observation made in Ref. [31], we find a discrepancy of $>3 \sigma$, even if possible unknown selection effects are accounted for. In fact we find that the single most extreme void expected within the SDSS survey volume does not produce an angle-averaged $\Delta T$ value within $3 \sigma$ of the observed average signal for 50 voids in G08a. On the other hand, in Ref. [36] Papai et al. perform a similar comparison using a different method, and report a discrepancy with $\Lambda$ CDM of only $\sim 2 \sigma$. As demonstrated in Section 4.2.1, this is not due to the slightly different form of the radial profile for superstructures used. Instead the source of the difference arises from the interpretation of the matched filter technique employed in Ref. [36].

Papai et al. calibrate their radial profiles against simulation to obtain a template for the ISW effect from structures. As they note, reconstructing accurate estimates of the ISW profiles from comparison with simulations of finite size is complicated by cosmic variance in the simulation, necessitating the use of a high-pass filter in the reconstruction. Once this filter is applied, their numerical template profiles agree well with our analytic ones. 
They then assume exactly the same template profile for all structures, scaled by choosing $\delta_{0}=1$ for superclusters and $\delta_{0}=-1$ for supervoids, and place these structures at redshift $z=0.52$ at the locations of the structures observed in G08a. However, this procedure does not account for the actual density contrast of structures that can be expected in $\Lambda$ CDM with gaussian primordial perturbations, nor does it account for the fact that not all structures will be identical. By contrast our analysis models the actual expected distribution of void depths and sizes. Although, as in our analysis, all structures are assumed to be centred at the same redshift for the purposes of calculating the template ISW shift for individual structures, when lines of sight overlap their template contributions are simply added.

In this way Papai et al. obtain the template ISW temperature map, scale this template map by a factor $\lambda$, and maximise the likelihood function

$$
L(\lambda)=-\frac{1}{2}\left(T_{\mathrm{CMB}}-\lambda T_{\mathrm{ISW}}\right) C^{-1}\left(T_{\mathrm{CMB}}-\lambda T_{\mathrm{ISW}}\right),
$$

where $T_{\mathrm{CMB}}$ is the WMAP ILC map, $T_{\mathrm{ISW}}$ is the constructed template ISW map and $C$ is the pixel covariance matrix obtained from best-fit $\Lambda \mathrm{CDM}$.

The value of $\lambda$ that maximises the likelihood $L(\lambda)$ can be interpreted as the average central density contrast in the superstructures [36]

$$
\lambda \approx \frac{1}{2}\left(\delta_{0}^{50 c}-\delta_{0}^{50 v}\right)
$$

where superscripts $50 c$ and $50 v$ refer to the average of 50 superclusters and 50 supervoids respectively (note that in the linear approximation voids and clusters are entirely equivalent except for a minus sign). This is merely the density contrast that the superstructures must have in order for the template to fit the data.

It is clear from the figures in Ref. [36] that the best-fit values are $\lambda>1$ for a wide range of radial scales of the structures. Thus, although the initial scenario, where all structures are postulated to have exactly $\left|\delta_{0}\right|=1$, is already extremely unlikely, in order to fit the observed CMB the template ISW map must be further scaled up by a factor $\lambda>1$. In this case the linear approximation used to calculate the template ISW map must break down (indeed it is not clear that the approximation can be valid even when $\left|\delta_{0}\right|=1$ ). Further note that physical voids are restricted to $\delta_{0}>-1$ making the interpretation of $\lambda>1$ even more problematic. Therefore although Ref. [36] find a reasonable fit for their template when $\lambda>1$, this value is indicative of a problem with modelling the observations. This qualitative conclusion is entirely compatible with the more careful quantitative calculation presented here.

Although this conclusion is not highlighted in Ref. [36] it is clear that correctly interpreted, both results in fact indicate a significant tension between the $\Lambda \mathrm{CDM}$ model of the ISW effect due to superstructures and the observation first reported in Ref. [31].

\section{$5 \quad$ Summary and prospectives}

We have calculated the integrated Sachs-Wolfe effect expected in $\Lambda$ CDM from $\sim 100 h^{-1} \mathrm{Mpc}$ size structures, using the density profiles predicted by the linear theory of gaussian perturbations [37]. We find that the most extreme superstructures in the SDSS volume will produce an ISW signal of $\lesssim 2 \mu \mathrm{K}$. This matches well with ISW maps generated from $N$-body simulations of the $\Lambda$ CDM cosmology [38]. 
Our result is about 3 times larger than earlier calculations [33, 34] which assumed compensated top-hat density profiles. Such an assumption, while well-motivated for nonlinear structures that have formed at small scales [40], should not apply on the large scales of the superstructures considered here. Ref. [35] noted that the ISW signal from such structures should therefore be larger and our results confirm this.

Nevertheless we have demonstrated that the ISW signal claimed to have been detected in Ref. [31] is still $>3 \sigma$ larger than the signal expected in $\Lambda$ CDM. This tension persists even after allowing for likely selection effects. In fact, even the most extreme underdensities in the SDSS volume would still produce a signal discrepant by $>3 \sigma$ with the observed signal. Therefore the observed signal cannot be due to a selection effect. We concur with Refs. $[33,34]$ that, if the observed signal is due to the ISW effect, deep superstructures appear to be far more numerous than expected in a $\Lambda \mathrm{CDM}$ cosmology.

This differs from the conclusion of Ref. [36] who also used similar density profiles. We have demonstrated that this difference is not due to the small difference in profiles used, and have argued that it is instead because those authors interpreted at face value their templatefitting method when applied for density contrasts where the template necessarily breaks down for voids (i.e., requires $\delta<-1$ !). A more appropriate interpretation is entirely compatible with the results presented here.

An interesting question is whether the expected signal of the most extreme superstructures in a $\Lambda \mathrm{CDM}$ universe is possible to detect in principle. For $504^{\circ}$ circles of the CMB, Ref. [31] reports the statistical uncertainty is $3.1 \mu \mathrm{K}$. Hence the expected statistical uncertainty in the average for a sample of $N$ such patches will be approximately $(3.1 \times \sqrt{50} / \sqrt{N}) \mu \mathrm{K}$, so that for $N \simeq 3000$, the uncertainty on a measurement of $\langle\Delta T\rangle$ is $\Delta T_{\text {noise }} \simeq 0.4 \mu \mathrm{K}$. Thus a detection of $|\langle\Delta T\rangle|=1 \mu \mathrm{K}$ can be made with roughly $2.5 \sigma$ significance, averaged over an ensemble of 3000 superstructures. From Fig. 2 it is seen that for the 3000 largest voids in the SDSS DR6 survey volume, $|\langle\Delta T\rangle| \sim 1 \mu \mathrm{K}$ which is of the right order of magnitude but somewhat too small for detection at high significance. However the SDSS window is not large enough to contain 3000 independent $4^{\circ}$ patches on the sky so in any case a larger survey would be needed in order to measure a statistically significant signal and this would contain more supervoids. This order-of-magnitude estimate indicates that even if the reported observation is a statistical anomaly or flawed in some other respect, the ISW imprint of superstructures in a $\Lambda$ CDM cosmology may be large enough to be detected in future surveys.

As noted earlier, the detection of the ISW imprint of individual superstructures provides an important complement to full-sky CMB-galaxy cross-correlation studies. It has the potential to provide information about the radii, density contrasts and density profiles of specific structures in the dark matter distribution that lie in the extreme tail of the probability distribution function. Our calculation demonstrates that the predicted ISW signal from the most extreme superstructures is far too small to explain the temperature fluctuations seen in Ref. [31], and that therefore the reported observations are in tension with the $\Lambda$ CDM model. Further observational investigation of this issue is warranted.

Nevertheless, the standard cosmology successfully fits many other observations, and any resolution of the discrepancy discussed here must also satisfy these constraints. A possible physical explanation would be that the primordial perturbations are non-gaussian. This would influence both the abundance of these extreme regions (e.g., [51, 52]) as well as their density profile, thus changing their expected ISW signal. A primordial non-gaussianity disproportionately affects the tails of the probability distribution function. Since the observation 
in Ref. [31] specifically probes the ISW effect of structures in these tails, it is expected to be more sensitive to non-gaussianity than the standard cross-correlation ISW studies. Interestingly, several cross-correlation studies also find an enhancement of the ISW signal over $\Lambda \mathrm{CDM}$ expectations, e.g. $[28,29,55]$, though given the large error bars this discrepancy has not been considered too significant. Therefore, primordial non-gaussianity may be able to explain this signal while preserving the success of $\Lambda \mathrm{CDM}$ on other fronts. However, this would then undermine the use of the ISW effect as an independent test for $\Lambda$.

It is worth noting that, qualitatively, a similar enhancement is found for overdensities as well as for voids, though we did not quantify this. As pointed in Ref. [53] a primordial skewness, parameterised by $f_{\mathrm{NL}}$, would not be able to enhance the abundance of both overand underdense regions simultaneously; however a primordial kurtosis, parameterised by a positive $g_{\mathrm{NL}}$, would indeed do so, and be less constrained by the CMB.

Another possible explanation might lie in a modification of the growth rate of perturbations as can happen in, e.g., models based on scalar-tensor gravity [54]. Such models may also change predictions by altering the behaviour of photons in gravitational potentials. The presence of large-scale inhomogeneities can themselves alter the growth rate and this too deserves further attention.

\section{Acknowledgements}

We would like to thank the anonymous referee for highlighting several areas where our arguments were unclear and for suggesting improvements. We thank Syksy Räsänen, Ben Hoyle and Tom Shanks for helpful comments and discussion, and Phil Bull for assistance with producing the figures. SH is supported by the Academy of Finland grant 131454 .

\section{References}

[1] R. Amanullah, C. Lidman, D. Rubin, G. Aldering, P. Astier, K. Barbary, M. S. Burns and A. Conley et al., Spectra and Light Curves of Six Type Ia Supernovae at $0.511<z<1.12$ and the Union2 Compilation, Astrophys. J. 716 (2010) 712 [arXiv:1004.1711 [astro-ph.CO]].

[2] A. Conley, J. Guy, M. Sullivan, N. Regnault, P. Astier, C. Balland, S. Basa and R. G. Carlberg et al., Supernova Constraints and Systematic Uncertainties from the First 3 Years of the Supernova Legacy Survey, Astrophys. J. Suppl. 192 (2011) 1 [arXiv:1104.1443 [astro-ph.CO]].

[3] M. Hicken, W. M. Wood-Vasey, S. Blondin, P. Challis, S. Jha, P. L. Kelly, A. Rest and R. P. Kirshner, Improved Dark Energy Constraints from 100 New CfA Supernova Type Ia Light Curves, Astrophys. J. 700 (2009) 1097 [arXiv:0901.4804 [astro-ph.CO]].

[4] E. Komatsu et al. [WMAP Collaboration], Seven-Year Wilkinson Microwave Anisotropy Probe (WMAP) Observations: Cosmological Interpretation, Astrophys. J. Suppl. 192 (2011) 18 [arXiv:1001.4538 [astro-ph.CO]].

[5] A. G. Riess, L. Macri, S. Casertano, H. Lampeitl, H. C. Ferguson, A. V. Filippenko, S. W. Jha and W. Li et al., A 3\% Solution: Determination of the Hubble Constant with the Hubble Space Telescope and Wide Field Camera 3, Astrophys. J. 730 (2011) 119 [Erratum-ibid. 732 (2011) 129] [arXiv:1103.2976 [astro-ph.CO]].

[6] B. A. Reid et al. [SDSS Collaboration], Baryon Acoustic Oscillations in the Sloan Digital Sky Survey Data Release 7 Galaxy Sample, Mon. Not. Roy. Astron. Soc. 401 (2010) 2148 [arXiv:0907.1660 [astro-ph.CO]]. 
[7] E. A. Kazin et al. [SDSS Collaboration], The Baryonic Acoustic Feature and Large-Scale Clustering in the SDSS LRG Sample, Astrophys. J. 710 (2010) 1444 [arXiv:0908.2598 [astro-ph.CO]].

[8] F. S. Labini, N. L. Vasilyev, Y. V. Baryshev and M. Lopez-Corredoira, Absence of anti-correlations and of baryon acoustic oscillations in the galaxy correlation function from the Sloan Digital Sky Survey DR7, Astron. Astrophys. 505 (2009) 981 [arXiv:0903.0950 [astro-ph.CO]].

[9] T. Biswas, A. Notari and W. Valkenburg, Testing the Void against Cosmological data: fitting $C M B, B A O, S N$ and HO, JCAP 1011 (2010) 030 [arXiv:1007.3065 [astro-ph.CO]].

[10] S. Nadathur and S. Sarkar, Reconciling the local void with the CMB, Phys. Rev. D 83 (2011) 063506 [arXiv:1012.3460 [astro-ph.CO]].

[11] R. K. Sachs and A. M. Wolfe, Perturbations of a cosmological model and angular variations of the microwave background, Astrophys. J. 147 (1967) 73 [Gen. Rel. Grav. 39 (2007) 1929].

[12] R. G. Crittenden and N. Turok, Looking for Lambda with the Rees-Sciama effect, Phys. Rev. Lett. 76 (1996) 575 [astro-ph/9510072].

[13] C. L. Bennett, R. S. Hill, G. Hinshaw, D. Larson, K. M. Smith, J. Dunkley, B. Gold and M. Halpern et al., Seven-Year Wilkinson Microwave Anisotropy Probe (WMAP) Observations: Are There Cosmic Microwave Background Anomalies?, Astrophys. J. Suppl. 192 (2011) 17 [arXiv:1001.4758 [astro-ph.CO]].

[14] N. Afshordi, Integrated Sachs-Wolfe effect in cross-correlation: The Observer's manual, Phys. Rev. D 70 (2004) 083536 [astro-ph/0401166].

[15] M. Douspis, P. G. Castro, C. Caprini and N. Aghanim, Optimising large galaxy surveys for ISW detection, Astron. Astrophys. 485 (2008) 395 arXiv:0802.0983 [astro-ph].

[16] S. Boughn and R. Crittenden, A correlation of the cosmic microwave sky with large scale structure, Nature 427 (2004) 45 [astro-ph/0305001].

[17] N. Afshordi, Y. -S. Loh and M. A. Strauss, Cross - correlation of the Cosmic Microwave Background with the 2MASS galaxy survey: Signatures of dark energy, hot gas, and point sources, Phys. Rev. D 69 (2004) 083524 [astro-ph/0308260].

[18] A. Cabre, E. Gaztanaga, M. Manera, P. Fosalba and F. Castander, Cross-correlation of wmap 3rd year and the sdss dr4 galaxy survey: new evidence for dark energy, Mon. Not. Roy. Astron. Soc. 372 (2006) L23 [astro-ph/0603690].

[19] P. Fosalba, E. Gaztanaga and F. Castander, Detection of the ISW and SZ effects from the CMB-galaxy correlation, Astrophys. J. 597 (2003) L89 [astro-ph/0307249].

[20] T. Giannantonio, R. G. Crittenden, R. C. Nichol, R. Scranton, G. T. Richards, A. D. Myers, R. J. Brunner and A. G. Gray et al., A high redshift detection of the integrated Sachs-Wolfe effect, Phys. Rev. D 74 (2006) 063520 [astro-ph/0607572].

[21] M. R. Nolta et al. [WMAP Collaboration], First year Wilkinson Microwave Anisotropy Probe (WMAP) observations: Dark energy induced correlation with radio sources, Astrophys. J. 608 (2004) 10 [astro-ph/0305097].

[22] N. Padmanabhan, C. M. Hirata, U. Seljak, D. Schlegel, J. Brinkmann and D. P. Schneider, Correlating the CMB with luminous red galaxies: The Integrated Sachs-Wolfe effect, Phys. Rev. D 72 (2005) 043525 [astro-ph/0410360].

[23] A. Raccanelli, A. Bonaldi, M. Negrello, S. Matarrese, G. Tormen and G. De Zotti, $A$ reassessment of the evidence of the Integrated Sachs-Wolfe effect through the WMAP-NVSS correlation, Mon. Not. Roy. Astron. Soc. 386 (2008) 2161 [arXiv:0802.0084 [astro-ph]].

[24] C. Hernandez-Monteagudo, Revisiting the WMAP - NVSS angular cross correlation: a skeptic 
view, Astron. Astrophys. 520 (2010) A101 [arXiv:0909.4294 [astro-ph.CO]].

[25] A. Rassat, K. Land, O. Lahav and F. B. Abdalla, Cross-correlation of 2MASS and WMAP3: Implications for the Integrated Sachs-Wolfe effect, Mon. Not. Roy. Astron. Soc. 377 (2007) 1085 [astro-ph/0610911].

[26] C. L. Francis and J. A. Peacock, ISW measurements with photometric redshift surveys: 2MASS results and future prospects, Mon. Not. Roy. Astron. Soc. 406 (2010) 2 [arXiv:0909.2494 [astro-ph.CO]].

[27] U. Sawangwit, T. Shanks, R. D. Cannon, S. M. Croom, N. P. Ross and D. A. Wake, Cross-correlating WMAP5 with 1.5 million LRGs: a new test for the ISW effect, Mon. Not. Roy. Astron. Soc. 402 (2010) 2228 [arXiv:0911.1352 [astro-ph.CO]].

[28] T. Giannantonio, R. Scranton, R. G. Crittenden, R. C. Nichol, S. P. Boughn, A. D. Myers and G. T. Richards, Combined analysis of the integrated Sachs-Wolfe effect and cosmological implications, Phys. Rev. D 77 (2008) 123520 [arXiv:0801.4380 [astro-ph]].

[29] S. Ho, C. Hirata, N. Padmanabhan, U. Seljak and N. Bahcall, Correlation of CMB with large-scale structure: I. ISW Tomography and Cosmological Implications, Phys. Rev. D 78 (2008) 043519 [arXiv:0801.0642 [astro-ph]].

[30] M. Lopez-Corredoira, F. S. Labini and J. Betancort-Rijo, Absence of significant cross-correlation between WMAP and SDSS, Astron. Astrophys. 513 (2010) A3 [arXiv:1001.4000 [astro-ph.CO]].

[31] B. R. Granett, M. C. Neyrinck and I. Szapudi, An Imprint of Super-Structures on the Microwave Background due to the Integrated Sachs-Wolfe Effect, Astrophys. J. 683 (2008) L99 arXiv:0805.3695 [astro-ph].

[32] B. R. Granett, M. C. Neyrinck and I. Szapudi, Dark Energy Detected with Supervoids and Superclusters, arXiv:0805.2974 [astro-ph].

[33] P. Hunt and S. Sarkar, Constraints on large scale inhomogeneities from WMAP-5 and SDSS: confrontation with recent observations, Mon. Not. Roy. Astron. Soc. 401 (2010) 547 [arXiv:0807.4508 [astro-ph]].

[34] K. T. Inoue, N. Sakai and K. Tomita, Evidence of Quasi-linear Super-Structures in the Cosmic Microwave Background and Galaxy Distribution, Astrophys. J. 724 (2010) 12 [arXiv:1005.4250 [astro-ph.CO]].

[35] P. Papai and I. Szapudi, Cosmological Density Fluctuations on 100Mpc Scales and their ISW Effect, Astrophys. J. 725 (2010) 2078 [arXiv:1009.0754 [astro-ph.CO]].

[36] P. Papai, I. Szapudi and B. R. Granett, Integrated Sachs-Wolf imprint of superstructures on linear scales, Astrophys. J. 732 (2011) 27 [arXiv:1012.3750 [astro-ph.CO]].

[37] J. M. Bardeen, J. R. Bond, N. Kaiser and A. S. Szalay, The Statistics of Peaks of Gaussian Random Fields, Astrophys. J. 304 (1986) 15.

[38] Y. -C. Cai, S. Cole, A. Jenkins and C. S. Frenk, Full-sky map of the ISW and Rees-Sciama effect from Gpc simulations, Mon. Not. Roy. Astron. Soc. 407, (2010) 201 arXiv:1003.0974 [astro-ph.CO].

[39] V. Springel, S. D. M. White, A. Jenkins, C. S. Frenk, N. Yoshida, L. Gao, J. Navarro and R. Thacker et al., Simulating the joint evolution of quasars, galaxies and their large-scale distribution, Nature 435 (2005) 629 [astro-ph/0504097].

[40] R. K. Sheth and R. van de Weygaert, A Hierarchy of voids: Much ado about nothing, Mon. Not. Roy. Astron. Soc. 350 (2004) 517 [astro-ph/0311260].

[41] O. Lahav, P. B. Lilje, Evolution of velocity and density fields around clusters of galaxies, Astrophys. J. 374, (1991) 29 
[42] A. Dekel, Astron. Astrophys. 101 (1981) 79

[43] J. K. Adelman-McCarthy et al. [SDSS Collaboration], The Sixth Data Release of the Sloan Digital Sky Survey, Astrophys. J. Suppl. 175 (2008) 297 [arXiv:0707.3413 [astro-ph]].

[44] M. C. Neyrinck, ZOBOV: a parameter-free void-finding algorithm, Mon. Not. Roy. Astron. Soc. 386 (2008) 2101 arXiv:0712.3049 [astro-ph].

[45] M. C. Neyrinck, N. Y. Gnedin and A. J. S. Hamilton, VOBOZ: An Almost - parameter - free halo - finding algorithm, Mon. Not. Roy. Astron. Soc. 356 (2005) 1222 [astro-ph/0402346].

[46] G. Hinshaw et al. [WMAP Collaboration], Five-Year Wilkinson Microwave Anisotropy Probe (WMAP) Observations: Data Processing, Sky Maps, and Basic Results, Astrophys. J. Suppl. 180 (2009) 225 [arXiv:0803.0732 [astro-ph]].

[47] A. Lewis, A. Challinor and A. Lasenby, Efficient computation of CMB anisotropies in closed FRW models, Astrophys. J. 538 (2000) 473 [astro-ph/9911177].

[48] K. N. Abazajian et al. [SDSS Collaboration], The Seventh Data Release of the Sloan Digital Sky Survey, Astrophys. J. Suppl. 182 (2009) 543 [arXiv:0812.0649 [astro-ph]].

[49] A. Lewis and S. Bridle, Cosmological parameters from CMB and other data: A Monte Carlo approach, Phys. Rev. D 66 (2002) 103511 [astro-ph/0205436].

[50] J. M. Colberg, R. K. Sheth, A. Diaferio, L. Gao and N. Yoshida, Voids in a lambda-CDM universe, Mon. Not. Roy. Astron. Soc. 360 (2005) 216 [astro-ph/0409162].

[51] M. Kamionkowski, L. Verde and R. Jimenez, The Void Abundance with Non-Gaussian Primordial Perturbations, JCAP 0901 (2009) 010 [arXiv:0809.0506 [astro-ph]].

[52] S. Matarrese, L. Verde and R. Jimenez, The abundance of high-redshift objects as a probe of non-Gaussian initial conditions, Astrophys. J. 541 (2000) 10 [astro-ph/0001366].

[53] K. Enqvist, S. Hotchkiss and O. Taanila, Estimating $f_{N L}$ and $g_{N L}$ from Massive High-Redshift Galaxy Clusters, JCAP 1104 (2011) 017 [arXiv:1012.2732 [astro-ph.CO]].

[54] R. Nagata, T. Chiba and N. Sugiyama, WMAP constraints on scalar-tensor cosmology and the variation of the gravitational constant, Phys. Rev. D 69 (2004) 083512 [astro-ph/0311274].

[55] T. Goto, I. Szapudi and B. R. Granett, Cross-correlation of WISE Galaxies with the Cosmic Microwave Background, Mon. Not. Roy. Astron. Soc. in press, arXiv:1202.5306 [astro-ph.CO] 